\title{
Depression bei COPD belastendste Komorbidität
}

Fragestellung: Welche Komorbiditäten beeinträchtigen COPDPatienten am meisten und beeinflussen den Verlauf negativ?

Hintergrund: COPD-Patienten leiden oft an mehreren Begleiterkrankungen. Es wurden einige Versuche unternommen, den Einfluss der Komorbiditäten auf die Lebensqualität und den Verlauf zu berechnen. Einzelne Indices identifizierten bis zu 12 Komorbiditäten, die die Mortalität dieser Patienten steigerten. Hier sollten nun die Komorbiditäten identifiziert werden, die den höchsten Einfluss auf Lebensqualität und Verlauf der COPD hatten (COMCOLD-

Frei A, Muggensturm P, Putcha $\mathrm{N}$ et al. Five comorbidities reflected the health status in patients with chronic obstructive pulmonary disease: the newly developed COMCOLD index. J Clin Epidemiol 2014;67(8):904-11.

Patienten und Methoden: In einer prospektiven Cross-sectionalen-Analyse wurden 408 Patienten mit COPD-GOLD
Die Patientenselbsteinschätzung erfolgte mit einer visuellen Analogskala von $0-100(0$ = todkrank, 100 = beste Gesundheit), zusätzlich wurde der Chronic Respiratory Questionnaire (CRQ) erhoben. Zur Erfassung von Angst und Depression wurde die Hospital Anxiety and Depression Scala verwendet.

Ergebnisse: Am häufigsten traten die Nebenerkrankungen Hypertonie 42,2\%, Arthrose 29,4 \%, Übergewicht 20,3\%, KHK/ Herzinsuffizienz 20,3\% auf. Nach Transformation in ein Regressionsmodel und Adjustierung der Komorbiditäten an die $\mathrm{FEV}_{1}$ (\%) ergab sich folgende Schweregradverteilung nach dem COMCOLD-Index (die Reihenfolge entspricht der Wertigkeit): Depression, Angststörung, pAVK, TIA, KHK/Herzinsuffizienz. II-IV mit 4 Wochen Exazerbationsfreiheit eingeschlossen.
Schlussfolgerungen: Die Depression hat den größten Einfluss auf die Gesundheit im Zusammenhang mit einer COPD. In der Praxis kann der COMCOLD-Index die Krankheitslast reflektieren und ermöglicht v.a. bei Forschungsvorhaben das Erfassen des Effekts von Komorbiditäten auf den Erfolg neuer Therapiemethoden.

\section{- Kommentar von Prof. Dr. med. Hans Jürgen Heppner}

\section{Komorbiditäten müssen mitbehandelt werden}

Kennzeichnend für geriatrische Patienten sind Multimorbidität und interindividuelle Heterogenität unter Berücksichtigung gesundheitsbezogener Veränderungen. Das hat Auswirkungen auf den Verlauf der COPD und die Exazerbationsraten. Relevante Komorbiditäten mit hoher Prävalenz sind gut beschrieben [1]. Die Erkrankungen beeinflussen sich wechselseitig negativ und führen im Verlauf zur Verschlechterung des Gesundheitszustands insgesamt. Diabetes mellitus als Komorbidität führt zu einer Verlängerung der Krankenhausverweildauer um 10\% und einer Steigerung der Sterblichkeit bei einer akuten Exazerbation um 8\% [2]. Die beiden Komorbiditäten Angststörung und/oder Depression treten in bis zu 70\% bei Patienten mit COPD auf und damit deutlich häufiger als in der Normalbevölkerung [3]. Das hat Folgen: Bei COPD mit Depression oder Angststörung sind Lebensqualität und körperliche Belastbarkeit vermindert COPD mit Depression oder Angststörung führt außerdem zu:

- häufigeren Exazerbationen,

- vermehrter Hospitalisierung,

- verlängerten Krankenhaus-Aufenthalten und

- erhöhter Sterblichkeit.

In einer Untersuchung fand sich in 30\% eine milde Demenz und in 22\% eine mittlere bis schwere Demenz als Begleiterkrankung [4] und die COPD ist mit kognitivem Abbau assoziiert. Dieser vollzieht sich schneller als in anderen Komorbiditätsgruppen [5]. Das Risiko für die Entwicklung eines M. Parkinson ist bei COPD 1,37-fach erhöht und damit deutlich über dem Risiko der Normalbevölkerung [6]. Auch die Osteoporose tritt bei COPD-Patienten mit 32,5\% deut- lich häufiger auf als normal (11,4\%) [7]. Bei der COPD-Therapie geht es nicht nur um die Verbesserung der Lungenfunktion, genauso wichtig sind Symptomkontrolle, Verringerung der Exazerbation [8] und die gezielte Therapie der Komorbiditäten. Komorbiditäten verringern die Lebensqualität, fördern die Exazerbation und steigern die Sterblichkeit und müssen daher behandelt werden.

Literatur:

1. Smith MC et al. International Journal of COPD 2014;9:871-888

2. Parappil A et al. Respirology 2010;15:918-22

3. Yohannes AM et al. Int J Geriatr Psychiatry. 2010;25:1209-1221

4. Frohnhofen H et al. Z Gerontol Geriat 2011;44:240-244

5. Zhou G et al. Dement Geriatr Cogn Disord Extra 2012;2:219-228

6. Li CH eta al. QJM 2015 Jan;108(1):39-45.

7. Graat-Verboom L et al. Eur Respir J 2009;34:209-218

8. Global strategy for the Diagnosis, Management and Prevention of COPD, Global Initiative for Chronic Obstructive Lung Disease (GOLD) 2015; www.goldcopd.org

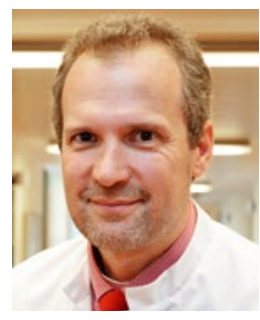

Prof. Dr. med. Hans Jürgen Heppner

Chefarzt Geriatrische Klinik und Tagesklinik Lehrstuhl für Geriatrie

Universität Witten/Herdecke

Dr.-Moeller-Straße 15, 58332 Schwelm hans-juergen.heppner@helios-kliniken.de hans.heppner@uni-wh.de 